\title{
Clones obtenidos de cruces dialélicos y de polinización libre de papas nativas seleccionados por estabilidad fenotípica*
}

\author{
Gustavo Javier Torres**
}

\section{RESUMEN}

Una muestra seleccionada de 24 clones $F_{1}$ de cruzas dialélicas y 33 clones $F_{1}$ de polinización libre fue evaluada por el grado de estabilidad fenotípica del rendimiento, y el número, tamaño y gravedad específica de tubérculos, en una serie de seis experimentos en diseño de bloques completos al azar conducidos en Huachac, El Mantara y Chuclú durante las campañas agrícolas 1989-90 y 1990-91.

El análisis de estabilidad fenotípica, sobre un rango de seis ambientes, muestra que el clon de polinización libre P21-38-0 fue simultáneamente estable en rendimiento (1.258 Kg./planta), en tamaño promedio de tubérculo (76.4 gr/planta) y en gravedad específica (1.094). Cuatro clones de polinización libre fueron simultáneamente ESTABLES en dos caracteres: el P09-07-8 en rendimiento y tamaño, el P13-59-9 en rendimiento y número de tubérculos por planta, el P01-11-0 y P02-01-0, ambos en número y gravedad específica. Estos clones serán nominados como nuevos cultivares nativos para ponerlos a disposición de los agricultores.

Se encontró 11 clones dialélicos y 11 clones de polinización libre estables en un solo carácter. Se discuten algunas implicaciones de este estudio en el mejoramiento genético de las papas nativas tetraploides andigena.

Palabras claves adicionales: Cultivares tetraploides Solanum tuberosum ssp. andigena; Parámetros de estabilidad fenotípica.

Aceptado para publicación: febrero 6, 1995

* Trabajo financiado (1989-91) por el Proyecto de T.T.A. de la Fundación para el Desarrollo del Agro (FUNDEAGRO), Av. Javier Prado Este 1894, San Borja, Lima - Perú.

** Ing. Agr. M. Sc., Profesor de la Escuela de Post Grado de la Universidad Nacional del Centro del Perú. Av. Calmell del Solar Nº 956, San Carlos, Huancayo - Perú. 


\section{SUMMARY}

\section{Clones Obtained From Diallel Crosses And From Open-Pollinated Native Potatoes Selected For Phenotypic Stability}

A selected sample of $24 \mathrm{~F}_{1}$ diallel hybryd clones and $33 \mathrm{~F}_{1}$ clones of openpollination were evaluated for the degree of phenotypic stability, yield, number, size and specific gravity of tubers in a series of six experiments based on a randomized complete block design, carried out at Huachac, El Mantaro and Chuclú during the 1989-90 and 1990-91 growing seasons.

The phenotypic stability analysis over a range of six environments showed that the open-pollinated clon P21-38-0 was simultaneously stable in yield (1.258 Kg./plant), average size tuber (76.4 gr/plant) and specific gravity (1.094). Four open-pollinated clones were simultaneously stables in two characters: the P09-07-8 in yield and size; the P13-59-9 in yield and number of tubers per plant; the P01-11-0 and P02-01-0, both at number and specific gravity. These clones will be released as new native cultivars to be used by farmers.

Eleven diallel and 11 open-pollinated clones were found to be stable in a single character. Some implications of this study on native andigena tetraploid potato breeding is discussed.

Additional index words: tetraploid native Solanum tuberosum ssp. andigema,; phenotypic stability parameters

El empleo de nuevas tecnologías para incrementar significativamente la producción y la productividad del cultivo de la papa de la región andina del Perú, implica desarrollar cultivares y técnicas de cultivo accesibles a las condiciones socioeconómicas de la gran mayoría de los pequeños agricultores.

Las crecientes migraciones a los centros urbanos a causa de la situación de aguda pobreza del agro, está produciendo una acelerada pérdida de cultivares nativos andinos que se caracterizan: por su gran tolerancia a los factores bióticos y abióticos adversos, por su alta estabilidad en la producción pese a las fuertes variaciones ambientales y al uso de tecnologías inadecuadas, y por su elevado valor nutritivo que alimenta a la población andina. 
Para dar alguna solución al problema de la erosión genética, en 1977 se propuso generar cultivares nativos andinos con características superiores, adecuadas a las necesidades socio-económicas de los pequeños agricultores, y que les permita incrementar su producción.

Utilizando la colección de papas nativas de la especie Solanum tuberosum ssp. andigena del Banco de Germoplasma de Papa que mantiene la EEA El Mantaro de la Universidad Nacional del Centro del Perú, se produjeron durante las campañas agrícolas de 1977-78 a 1984-85, paralela y simultáneamente, dos poblaciones de clones híbridos $\mathrm{F}_{1}$ : 3,646 derivados de cruzas dialélicas en diseño 3 entre 15 y entre 10 cultivares; y 4,124 derivados de polinización libre de 23 a 51 cultivares nativos andigena.

Ambas poblaciones de clones agrupadas en 8 subpoblaciones por el año de cruzamiento (1978 a 1985), fueron sometidas a la presión de selección natural durante tres a diez campañas agrícolas. Cada subpoblación formada por clones dialélicos y de polinización libre constituyó un experimento de evaluación preliminar que fue conducido con mediana fertilización y escasa aplicación de pesticidas.

Mediante estos experimentos, repetidos en un sólo lugar (EEA El Mantaro), se efectuó parte del proceso de eliminación de los clones segregantes indeseables con el objeto primario de identificar clones $F_{1}$, cuyo promedio sobre los años fuese alto y estable. Como resultado de la selección natural y del proceso de eliminación referido, en la campaña agrícola 1988-89 las poblaciones se redujeron a 1,030 clones dialélicos y 834 clones de polinización libre.

Para identificar los "mejores" clones por su estabilidad fenotípica en cuatro caracteres mediante la prueba en varios lugares, dichas poblaciones previamente fueron sometidas a un proceso de selección individual por seis caracteres: sobrevivencia de plantas, rendimiento, forma de tubérculo, profundidad de ojos, color de la carne y de la piel, seleccionándose 370 clones dialélicos y 274 clones de polinización libre. Luego se hizo una reselección por su rendimiento durante tres años, resultando seleccionados 77 dialélicos y 76 de polinización libre.

Con el fin de seleccionar nuevos cultivares de papas nativas andigena para ponerlos a disposición de los pequeños y medianos productores de la región andina del Perú, dichos clones fueron evaluados por el grado de estabilidad fenotípica en cuatro caracteres en una serie de tres experimentos en Huachac, El Mantaro y en Chuclú-Jauja durante la campaña agrícola 1989-90; encontrándose 7 y 41 clones fenotípicamente estables en dos y un caracteres, respectivamente. 
Para verificar los resultados de los 48 clones, en la campaña agrícola 1990-91 se repitió la serie de experimentos en los mismos tres lugares, agregándose 9 clones de la serie anterior y 36 clones no probados de las poblaciones originales.

El presente artículo se refiere a los resultados obtenidos con los 57 clones (24 dialélicos y 33 de polinización libre) que fueron evaluados en seis condiciones ambientales diferentes mediante seis experimentos conducidos durante las campañas agrícolas de 1989-90 y 1990-91; con los objetivos siguientes:

a) Evaluar el grado de estabilidad fenotípica del rendimiento, número, tamaño y gravedad específica de tubérculos de 24 clones $F_{1}$ dialélicos y 33 clones $F_{1}$ de polinización libre comparado con 7 clones mejorados, como controles.

b) Seleccionar clones $F_{1}$ con rendimientos altos y estables a un amplio espectro de variaciones ambientales, en dos o más caracteres económicos, de buena forma de tubérculos, de buen color de carne y de buena calidad nutritiva, para nominarlos como nuevos cultivares nativos andigena y ponerlos a disposición de los agricultores para que sean cultivados individualmente o en mezclas.

\section{MATERIALES Y MÉTODOS}

\section{Series de Experimentos}

Durante las campañas agrícolas 1989-90 y 1990-91 se condujeron en Huachac (3,353 m), EEA ELMantaro (3,316 m) y en Chuclú-Jauja (3,370 m), dos series de tres experimentos. Estos lugares representan el área geográfica del Valle Mantaro en una longitud de $50 \mathrm{~km}$.

En la serie de tres experimentos de 1989-90 se probaron 77 clones híbridos $\mathrm{F}_{1}$ dialélicos (D), 76 clones F, de polinización libre (P) y 7 cultivares control: Bolívar, Sucre, Grau, Bolognesi, Constitución, Huaycha y Ollanta, en la serie de tres experimentos de 1990-91 intervinieron 24 D y 33 P probados en 1989-90; 22 D y 14 P adicionales, y los 7 cultivares control indicados.

En efecto, se trató de seleccionar de entre los 24 D y 33 P, aquellos clones fenotípicamente más estables en dos o más caracteres, en respuesta a las seis condiciones ambientales diferentes a que fueron sometidos en las dos últimas campañas, después de un proceso continuado de selección natural y artificial durante 13 años, como se muestra en el esquema de la Figura 1. 


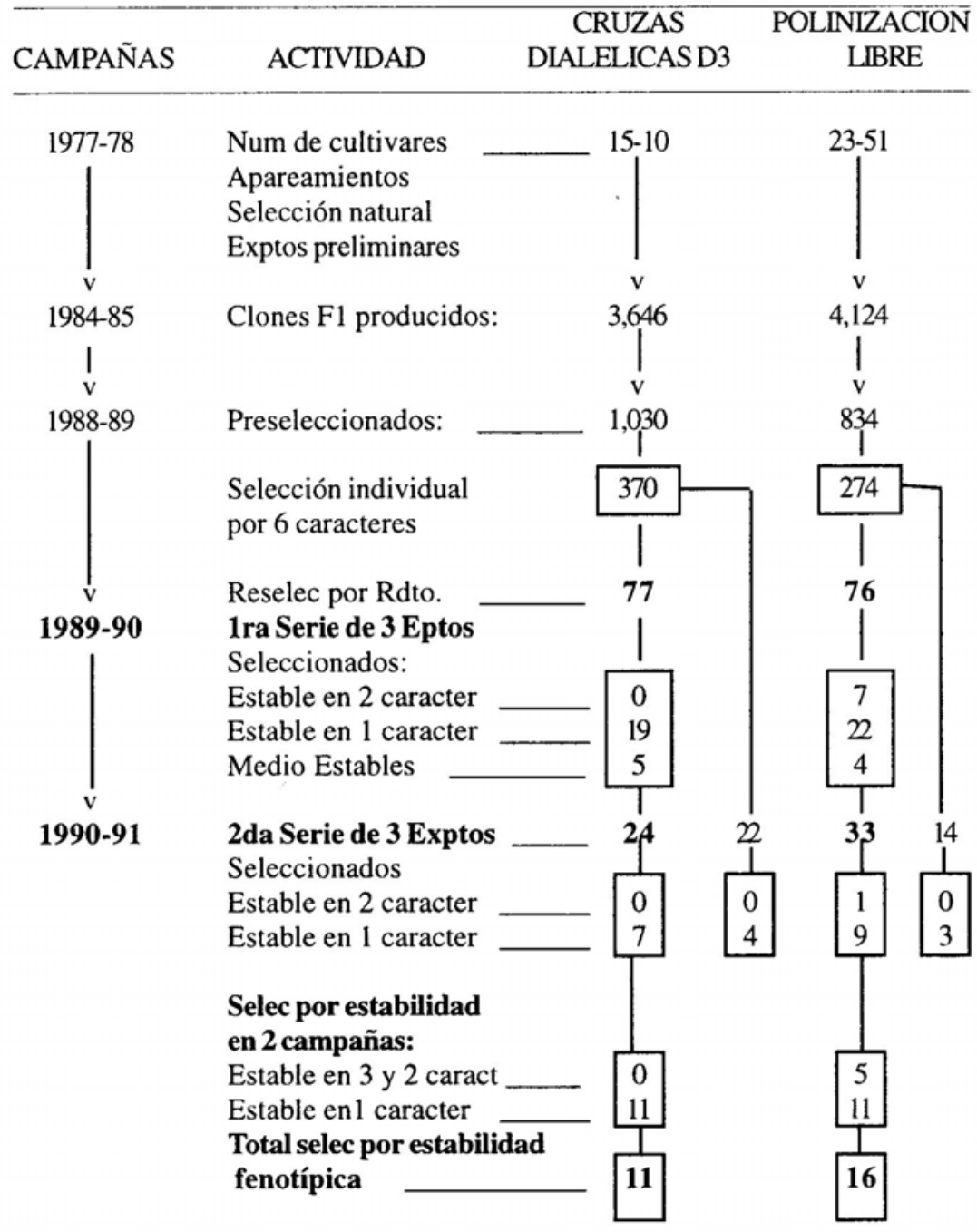

Fig. 1 Secuencia seguida en el desarrollo de clones $F_{1}$ a partir de Cruzas Dialélicas en diseño 3 y de Polinización Libre.

Cada uno de los experimentos de las series se basó en un diseño de bloques completos al azar con dos bloques subdivididos en 5 sub-bloques. Parcelas de un surco de $2 \mathrm{~m}$ de largo. Se sembraron cinco tubérculos por parcela a la densidad de $0.4 \mathrm{~m}$ entre plantas y $1 \mathrm{~m}$ entre surcos. La fórmula de abonamiento NPK utilizada fue de 160-140-120. El control de plagas y enfermedades se redujo al mínimo. 
En la campaña agrícola de 1989-90 los tres experimentos soportaron los efectos de la sequía, heladas y granizadas que dañaron en diversos grados a los clones, mostrándose muchos de ellos como muy tolerantes. Y en la campaña agrícola de 1990-91, los tres experimentos soportaron los efectos del exceso de lluvias ocurridas al inicio y al final del desarrollo vegetativo, causando la pudrición de tubérculos en cierto número de clones.

\section{Caracteres analizados}

a) Rendimiento por planta en kg.

b) Número de tubérculos por planta.

c) Peso promedio de tubérculo por planta en g como medida del tamaño.

d) Gravedad específica de tubérculos, calculado por el cociente:

G.e.= (Peso en aire - C)/(Peso en aire - C - Peso en agua) Donde, $\mathrm{C}$ es el peso de la canastilla en el aire y sumergida; su peso fue nivelado a cero.

La gravedad específica se determinó tomando una muestra de 400 a 900 g de tubérculos de cada parcela después de una semana de cosechados.

\section{Análisis de Estabilidad Fenotípica:}

Previamente al análisis de estabilidad fenotípica, los datos de la serie de seis experimentos correspondientes a los 4 caracteres de los 57 clones probados y de los 7 controles fueron sometidos a un análisis combinado de la varianza mediante el modelo matemático clásico.

El análisis de estabilidad fenotípica de los 4 caracteres de los 64 clones se hizo utilizando el modelo de estabilidad (1).

La determinación del grado de estabilidad fenotípica de un carácter de los clones se hizo mediante el diagrama de dispersión de los puntos determinados por la intersección de la media genotípica y el coeficiente de regresión de cada clon (6).

\section{RESULTADOS}

La Tabla 1 muestra que en los cuatro caracteres, existen diferencias altamente significativas entre los efectos medios de los clones y entre las respuestas de los clones al efecto de las variaciones ambientales que ocurrieron en los tres lugares en las dos campañas agrícolas. Este último efecto esta expresado por la alta significación de la interacción clones x ambientes. 
Tabla 1. Análisis combinado de la varianza de 4 caracteres de 64 clones probados en 6 ambientes.

\begin{tabular}{lrcccc}
\hline \multicolumn{6}{c}{ Cuadrados Medios } \\
\hline FV & GL & $\begin{array}{c}\text { Rdto } \\
\text { kg/plt }\end{array}$ & $\begin{array}{c}\text { Número } \\
\text { Tub/plt }\end{array}$ & $\begin{array}{c}\text { Tamaño } \\
\text { g/plt }\end{array}$ & $\begin{array}{c}\text { Gravedad } \\
\text { específica }\end{array}$ \\
\hline Ambientes & 5 & 8.779 & 789.79 & 17050.9 & 0.01285 \\
Rep/Ambientes & 6 & 0.328 & 188.90 & 1009.8 & 0.00005 \\
Clones & 63 & $0.418^{* *}$ & $401.51^{* *}$ & $5479.2 * *$ & $0.00075^{* *}$ \\
CLxAmb. & 315 & $0.185^{* *}$ & $61.96^{* *}$ & $758.4^{* *}$ & $0.00016^{* *}$ \\
Error Comb. & 378 & 0.093 & 41.94 & 444.0 & 0.00007 \\
Total & 767 & & & & \\
\hline Media General & & 0.834 & 16.6 & 57.2 & 1.087 \\
CV\% & 1 & 36.5 & 39.0 & 36.8 & 0.8
\end{tabular}

** Significativo al nivel del $1 \%$.

Los índices ambientales son como una medida de la productividad de cada uno de los caracteres bajo las condiciones en que se desarrollaron los clones en las dos campañas agrícolas.

Tabla 2. Indices ambientales estimados de los 6 ambientes correspondientes a los 4 caracteres.

\begin{tabular}{lcccc}
\hline & \multicolumn{4}{c}{ Indices Ambientales } \\
\cline { 2 - 5 } & $\begin{array}{c}\text { Rdto } \\
\mathrm{kg} / \mathrm{plt}\end{array}$ & $\begin{array}{c}\text { Número } \\
\text { Tub/plt }\end{array}$ & $\begin{array}{c}\text { Tamaño } \\
\text { g/plt }\end{array}$ & $\begin{array}{c}\text { Gravedad } \\
\text { específica }\end{array}$ \\
\hline Ambientes & & & & \\
Huachac 89-90 & 0.213 & 0.414 & 14.560 & -0.0034 \\
Mantaro 89-90 & 0.425 & 3.703 & 10.771 & 0.0043 \\
Chuclú 89-90 & -0.248 & 1.830 & -17.406 & 0.0057 \\
Huachac 90-91 & -0.132 & -1.576 & -5.009 & -0.0085 \\
Mantaro 90-91 & -0.082 & -1.203 & 0.233 & -0.0126 \\
Chuclú 90-91 & -0.176 & -3.169 & -3.149 & 0.0144 \\
\hline
\end{tabular}

La Tabla 3 muestra que entre las medias genotípicas de los clones existen diferencias altamente significativas para los cuatro caracteres; y que entre los clones existen diferencias genéticas altamente significativas por su regresión sobre los índices ambientales en el rendimiento, tamaño y gravedad específica de tubérculos. No existen diferencias genéticas (CL x Amb Lin) significativas en el número de tubérculos por planta. 
Tabla 3. Análisis de la varianza de la estabilidad fenotípica de 4 caracteres de 64 clones sobre 6 ambientes.

\begin{tabular}{|c|c|c|c|c|c|}
\hline & & & Cuadrados & Medios & \\
\hline FV & GL & $\begin{array}{l}\text { Rdto } \\
\mathrm{kg} / \mathrm{plt}\end{array}$ & $\begin{array}{c}\text { Número } \\
\text { Tub/plt }\end{array}$ & $\begin{array}{c}\text { Tamaño } \\
\text { g/plt }\end{array}$ & $\begin{array}{l}\text { Gravedad } \\
\text { específica }\end{array}$ \\
\hline Clones & 6 & $0.209 * *$ & $200.76^{* *}$ & $2739.6 * *$ & $0.00037 * * *$ \\
\hline Amb Lineal & & 21.950 & 1997.00 & 42627.4 & 0.03217 \\
\hline $\mathrm{Cl} \times \mathrm{Amb}$ Lin & 6 & $0.140 * *$ & 36.18 & $629.9 * *$ & $0.00013^{* *}$ \\
\hline Desv Comb. & 25 & 0.079 & 29.22 & 311.5 & 0.00007 \\
\hline Total & 38 & & & & \\
\hline CV\% & & 33.7 & 32.6 & 30.9 & 0.8 \\
\hline ** Significat & tivo al nive & l del $1 \%$. & & & \\
\hline $\begin{array}{r}\text { Tabla 4. Pa } \\
\text { fen } \\
\text { sim }\end{array}$ & $\begin{array}{l}\text { arámetro. } \\
\text { otípicame } \\
\text { uultáneam }\end{array}$ & $\begin{array}{l}\text { de estabilid } \\
\text { nte ESTABL } \\
\text { ente. }\end{array}$ & $\begin{array}{l}\text { idad estimad } \\
\text { LES y MUY }\end{array}$ & $\begin{array}{l}\text { os de } 5 \text { clone } \\
\text { ESTABLES }\end{array}$ & $\begin{array}{l}\text { que resultaro } \\
3 \text { y } 2 \text { caracter }\end{array}$ \\
\hline Clon & Carácter & Ecuación de & le Regr. & Regr. & Desv Regr \\
\hline P21-38-0 & Rdto & $Y=1.258+$ & $+0.749 \mathrm{I}$ & 0.0851 & 0.0384 \\
\hline & Tamaño & $\mathrm{Y}=76.4+$ & $+0.313 \mathrm{I}$ & 545.46 & 323.45 \\
\hline & Grav esp & $\mathrm{Y}=1.094+$ & $+1.102 \mathrm{I}$ & 0.00009 & 0.00005 \\
\hline P09-07-8 & Rdto & $\mathrm{Y}=1.238+$ & + $1.374 \mathrm{I}$ & 0.1970 & 0.1504 \\
\hline & Tamaño & $Y=76.4+$ & $+0.216 \mathrm{I}$ & 447.00 & 224.99 \\
\hline P13-59-9 & Rdto & $Y=1.089+$ & + 1.339 I & 0.0697 & 0.0230 \\
\hline & Número & $\mathrm{Y}=30.7+$ & $+0.422 \mathrm{I}$ & 125.45 & 104.48 \\
\hline P0l-11-0 & Número & $Y=21.8+$ & +1.292 I & 13.111 & -7.861 \\
\hline & Grav esp & $Y=1.102-$ & 0.112 I & 0.00008 & 0.00005 \\
\hline P02-01-0 & Número & $\mathrm{Y}=23.2+$ & $+2.141 \mathrm{I}$ & 15.021 & -5.951 \\
\hline & & $\mathrm{Y}=1.102-$ & $-0.456 \mathrm{I}$ & 0.00009 & 0.00006 \\
\hline
\end{tabular}


Tabla 5. Estimadores de la media genotípica, del coeficiente de regresión y de los límites de confianza del $95 \%$ basado en la distribución de $t$ (=1.98 a 0.05 con 63 g.L), utilizados para la determinación de los grados de estabilidad fenotípica de 4 caracteres de 64 clones.

\begin{tabular}{lcccc}
\hline Estimadores & $\begin{array}{c}\text { Rdto } \\
\mathrm{kg} / \mathrm{plt}\end{array}$ & $\begin{array}{c}\text { Número } \\
\text { Tub/plt }\end{array}$ & $\begin{array}{c}\text { Tamaño } \\
\text { g/plt }\end{array}$ & $\begin{array}{c}\text { Gravedad } \\
\text { específica }\end{array}$ \\
\hline Media Genotípica & & & & \\
Media general & 0.834 & 16.6 & 57.2 & 1.087 \\
Error estándar & 0.126 & 2.417 & 7.894 & 0.004 \\
L inferior del IC & 0.585 & 11.8 & 41.6 & 1.079 \\
L superior del IC & 1.084 & 21.4 & 72.8 & 1.094 \\
Coef de Regresión & & & & \\
Media general & 1.0 & 1.0 & 1.0 & 1.0 \\
Error estándar & 0.481 & 0.968 & 0.684 & 0.364 \\
L inferior del IC & 0.047 & -0.916 & -0.354 & 0.280 \\
L superior del IC & 1.953 & 2.916 & 2.354 & 1.720 \\
\hline
\end{tabular}

L del IC = Límite del intervalo de confianza de 95\%

Tabla 6. Número de clones $F_{1}$ dialélicos $(D)$ y de polinización libre (P) por el grado de estabilidad fenotípica dentro de cada carácter, en respuesta al rango de variaciones de 6 ambientes, del total de 57 clones.

\begin{tabular}{lcccccccc}
\hline Grados de Estabilidad & \multicolumn{3}{c}{ Rdto } & \multicolumn{1}{c}{ Número } & \multicolumn{3}{c}{ Tamaño } & \multicolumn{2}{c}{ Grav esp. } \\
\cline { 2 - 9 } Fenotípica & $\mathrm{D}$ & $\mathrm{P}$ & $\mathrm{D}$ & $\mathrm{P}$ & $\mathrm{D}$ & $\mathrm{P}$ & $\mathrm{D}$ & $\mathrm{P}$ \\
\hline MUY ESTABLE & 0 & 0 & 1 & 0 & 0 & 0 & 0 & 2 \\
& 1 & 3 & 1 & 5 & 4 & 8 & 4 & 4 \\
ESTABLE & 0 & 1 & 1 & 2 & 1 & 0 & 0 & 0 \\
INESTABLE & 1 & 1 & 0 & 0 & 1 & 0 & 1 & 2 \\
MEDIO MUY ESTABLE & 18 & 22 & 13 & 18 & 11 & 13 & 12 & 17 \\
MEDIO ESTABLE & 2 & 0 & 2 & 1 & 1 & 2 & 3 & \\
MEDIO MUY INESTABLE0 & 4 & 4 & 8 & 6 & 6 & 11 & 5 & 5 \\
INDESEABLE & & &
\end{tabular}

La Tabla 7 muestra que de los 57 clones $F_{1}$ sólo un clon presenta 3 caracteres simultáneamente estables y cuatro clones presentan 2 caracteres simultáneamente estables, siendo los cinco derivados de polinización libre. 
Los clones restantes, correspondientes a 11 dialélicos y 11 de polinización libre, presentan sólo un carácter estable. Se muestra también el grado de estabilidad de los otros caracteres de cada uno de los clones; observándose que los clones tienen diferentes grados de estabilidad en sus diversos caracteres.

Tabla 7. Clones $F_{1}$ con 3, 2 y 1 caracteres fenotípicamente ESTABLES (E) y MUY ESTABLES (ME) simultáneamente y grados de estabilidad de los otros caracteres.

\begin{tabular}{|c|c|c|c|c|}
\hline Clones $\mathrm{F}_{1}$ & $\begin{array}{l}\text { Rdto } \\
\text { kg/plt }\end{array}$ & $\begin{array}{l}\text { Número } \\
\text { tub/plt }\end{array}$ & $\begin{array}{l}\text { Tamaño } \\
\text { g/plt }\end{array}$ & $\begin{array}{l}\text { Gravedad } \\
\text { específica }\end{array}$ \\
\hline P21-38-0 & $1.258 \mathrm{E}$ & $17.5 \mathrm{mE}$ & $76.4 \mathrm{E}$ & $1.094 \mathrm{E}$ \\
\hline P09-07-8 & $1.238 \mathrm{E}$ & $17.5 \mathrm{mE}$ & $76.4 \mathrm{E}$ & $1.082 \mathrm{mE}$ \\
\hline P13-59-9 & $1.089 \mathrm{E}$ & 30.7E & 38.1ID & 1.079ID \\
\hline P01-11-0 & $0.687 \mathrm{mE}$ & $21.8 \mathrm{E}$ & 31.0ID & $1.102 \mathrm{ME}$ \\
\hline P02-01-0 & $0.795 \mathrm{mE}$ & $23.2 \mathrm{E}$ & 33.2ID & $1.102 \mathrm{ME}$ \\
\hline D10-03-8 & $0.800 \mathrm{mE}$ & 10.0ID & $86.0 \mathrm{E}$ & 1.079ID \\
\hline D16-12-8 & $1.176 \mathrm{E}$ & 17.3mE & $70.1 \mathrm{mE}$ & 1.080mMI \\
\hline D28-02-9 & $0.782 \mathrm{mE}$. & $18.2 \mathrm{mE}$ & $42.6 \mathrm{mE}$ & $1.098 \mathrm{E}$ \\
\hline P04-50-9 & $1.013 \mathrm{mE}$ & $12.9 \mathrm{mE}$ & 81.7E & $1.090 \mathrm{mE}$ \\
\hline P31-50-9 & 0.585 ID & $17.7 \mathrm{mE}$ & 36.6ID & $1.095 \mathrm{E}$ \\
\hline D24-11-0 & $0.851 \mathrm{mE}$ & $180 \mathrm{mE}$ & $49.9 \mathrm{mE}$ & $1.094 \mathrm{E}$ \\
\hline P02-02-0 & $0.723 \mathrm{mE}$ & $17.5 \mathrm{mE}$ & $44.7 \mathrm{mE}$ & $1.098 \mathrm{E}$ \\
\hline P12-05-0 & $1.026 \mathrm{mMI}$ & $12.2 \mathrm{mE}$ & $76.8 \mathrm{E}$ & 1.079ID \\
\hline P13-28-0 & $1.050 \mathrm{mE}$ & $21.8 \mathrm{E}$ & $50.2 \mathrm{mE}$ & $1.092 \mathrm{mE}$ \\
\hline D09-05-2 & $0.959 \mathrm{mE}$ & 23.21 & $48.4 \mathrm{mE}$ & $1.094 \mathrm{E}$ \\
\hline P06-03-2 & $0.762 \mathrm{mE}$ & 10.1ID & $76.1 \mathrm{E}$ & $1.089 \mathrm{mE}$ \\
\hline P22-03-2 & $0.793 \mathrm{mE}$ & 9.1ID & 106.8E & 1.082mME \\
\hline D13-42-3 & $0.815 \mathrm{mE}$ & 10.5ID & $79.7 \mathrm{E}$ & $1.092 \mathrm{mE}$ \\
\hline D18-21-3 & $0.972 \mathrm{mE}$ & $12.0 \mathrm{mE}$ & $82.2 \mathrm{E}$ & $1.072 \mathrm{ID}$ \\
\hline D41-91-3 & $0.819 \mathrm{mE}$ & $14.1 \mathrm{mE}$ & $60.2 \mathrm{mE}$ & $1.096 \mathrm{E}$ \\
\hline P12-16-3 & $0.981 \mathrm{mE}$ & 11.2ID & 87.3E & $1.085 \mathrm{mE}$ \\
\hline P13-03-3 & $0.949 \mathrm{mE}$ & $29.8 \mathrm{E}$ & 31.5ID & 1.079ID \\
\hline P25-19-3 & $0.622 \mathrm{mE}$ & $12.5 \mathrm{mE}$ & $49.2 \mathrm{mE}$ & $1.104 \mathrm{E}$ \\
\hline P36-07-3 & $0.748 \mathrm{mE}$ & 9.1ID & $79.1 \mathrm{E}$ & 1.073ID \\
\hline D13-11-5 & $0.719 \mathrm{mE}$ & $26.6 \mathrm{E}$ & 26.6ID & $1.083 \mathrm{mE}$ \\
\hline D13-13-5 & 0.712mME & 21.7ME & 33.4ID & 1.071ID \\
\hline D19-06-5 & $0.743 \mathrm{mE}$ & 8.8ID & $92.5 \mathrm{E}$ & $1.085 \mathrm{mE}$ \\
\hline
\end{tabular}
mME = MEDIO MUY ESTABLE; mE=MEDIO ESTABLE; I = INESTABLE; mMI = MEDIO MUY INESTABLE; ID = INDESEABLE 


\section{DISCUSIÓN}

Los valores de los índices ambientales (Tabla 2), expresan que el rango de variaciones de los factores ambientales de las dos campañas agrícolas en los tres lugares, produjeron una interacción genotipo-ambiente altamente significativa (Tablas 1 y 3), que influyó en la productividad de los cuatro caracteres de los clones.

La alta significación de la interacción clones $\mathrm{x}$ ambiente lineal indica que entre los clones existen diferencias genéticas altamente significativas por su respuesta al efecto de las variaciones ambientales, expresado por los índices ambientales (Tablas 3).

Una variedad con una media alta, un coeficiente de regresión igual a la unidad y una varianza de las desviaciones desde la regresión no significativamente diferente de cero, ha sido definida como una variedad (genotipo) estable, por (1).

Pero desde el punto de vista de la producción agrícola bajo las condiciones ambientales de la región de los Andes, dicha definición no es satisfactoria, puesto que se requiere de cultivares que presenten valores genotípicos de dos o más caracteres, significativamente superiores al promedio de un conjunto de cultivares sobre un rango de ambientes, y que respondan paralelamente a los efectos ambientales. Esto significa que se debe seleccionar clones con un coeficiente de regresión tendiente a cero, una varianza de las desviaciones desde la regresión significativamente igual a cero y un valor genotípico del carácter significativamente superior a la media genotípica general sobre una serie de ambientes.

En efecto, basado en estos criterios se efectuó el proceso de selección (Tabla 5), en la que, la determinación del grado de estabilidad fenotípica de un carácter se hizo mediante la dispersión de los puntos determinados por la intersección de la media genotípica y el coeficiente de regresión del clon en un espacio bidimensional, subdividido en 9 cuadrantes por líneas a partir de los límites del intervalo de confianza de $95 \%$ de las respectivas medias generales, en donde se han definido siete grados de estabilidad fenotípica (6 y 7).

Esta técnica estadística se basa en (1), quienes señalan que en la selección de híbridos estables, es útil usar un gráfico de puntos determinados por la relación entre el rendimiento promedio y el coeficiente de regresión de los híbridos; subdividiendo el espacio muestral por líneas verticales y horizontales a una desviación estándar respectivamente, de la media general y de la media de la pendiente. 
El clon P21 -38-0 con 3 caracteres simultáneamente ESTABLES (Tabla 4), presenta un coeficiente de regresión y la varianza de las desviaciones de regresión estadísticamente iguales a cero. Igualmente los 4 clones con 2 caracteres simultáneamente ESTABLES, presentan ambos parámetros estadísticamente iguales a cero o sólo uno de ellos. En ningún caso ambos son estadísticamente mayores que cero.

La distribución de frecuencia del número de clones $F_{1}$ dialélicos (D) y de polinización libre (P) (Tabla 6), muestra que en el conjunto de 57 clones, ocurrieron en diferentes proporciones los 7 grados de estabilidad fenotípica:

Clones MUY ESTABLES escasos en número y gravedad específica, y no existen en rendimiento y tamaño de tubérculos.

Clones ESTABLES se encontraron pocos en los 4 caracteres, con mayor frecuencia en los clones $\mathrm{P}$.

Clones INESTABLES muy escasos en los 3 caracteres, y en gravedad específica no se encontró ninguno.

Clones MEDIO MUY ESTABLES muy escasos en los 3 caracteres, y en número no se encontró ninguno.

Clones MEDIO ESTABLES notablemente frecuentes en los 4 caracteres y con predominancia de los clones $\mathrm{P}$.

Clones MEDIO MUY INESTABLES escasos en los 4 caracteres.

Clones INDESEABLES relativamente frecuentes en los 4 caracteres tanto en los clones D y P.

La selección simultánea por los 4 caracteres mediante la determinación del grado de estabilidad fenotípica de cada carácter en forma independiente, condujo a la identificación de tres grupos de clones $\mathrm{F}_{1}$ con 3,2 y 1 caracteres fenotípicamente estables (Tabla 7).

El clon P21 -38-0, ESTABLE simultáneamente en rendimiento, tamaño y gravedad específica, y MEDIO ESTABLE en número de tubérculos, es el mejor. Con tales características de estabilidad o adaptabilidad a las variaciones ambientales que incluyen los estreses bióticos y abióticos, constituye un clon de alto valor económico y de gran comportamiento bajo las condiciones ambientales de la región de los Andes. Por lo tanto será lanzado como un nuevo cultivar nativo tetraploide andigena para su utilización por los agricultores de subsistencia y empresariales.

Del grupo de cuatro clones estables en 2 caracteres, el clon P09-07-8, ESTABLE en rendimiento y tamaño de tubérculos, y MEDIO ESTABLE en número de tubérculo y gravedad específica, es el mejor. Los tres restantes, aunque INDESEABLES en tamaño de tubérculos, pueden constituir nuevos 
cultivares, especialmente los clones P01-11-0 y P02-01-0, ESTABLES en número y gravedad específica, y MEDIO ESTABLE en rendimiento. En cambio, el P13-59-9, aunque ESTABLE en rendimiento y número, tiene el inconveniente de ser INESTABLE en tamaño y gravedad específica.

Del grupo de 22 clones $F_{1}$ dialélicos y de polinización libre, estables en un sólo carácter, siete de ellos podrían constituirse en nuevos cultivares puesto que son MEDIO ESTABLES en los tres caracteres restantes.

En relación al origen de los clones $\mathrm{F}_{1}$ se observa que los clones con mayor número de caracteres estables se derivan de polinización libre: cinco clones con 3 y 2 caracteres estables y once con un sólo carácter estable (Tabla 7).

Se concluye que de una población de 3,646 clones $F_{1}$ dialélicos y 4,124 clones $F_{1}$ de polinización libre (Figura 1), sometida a un intenso proceso de selección natural y artificial en experimentos preliminares; a la selección individual por seis caracteres con reselección por rendimiento; y a la selección por estabilidad fenotípica de cuatro caracteres en seis ambientes; finalmente se seleccionaron 11 clones dialélicos y 16 clones de polinización libre.

De estos resultados se infiere que en el desarrollo de nuevos cultivares nativos tetraploides andigena, el método de apareamiento por polinización libre, genéticamente es tan eficaz o probablemente mejor que el método de apareamiento por cruzas dialélicas.

Dicha inferencia apoya la hipótesis de que, debido al alto grado de heterozigosidad de los cultivares nativos tetraploides andigena, las progenies de polinización libre son muy variables; $\mathrm{y}$ que mediante el proceso de selección natural y artificial a que fueron sometidos, se eliminaron muchos genotipos endogámicos con caracteres indeseables, quedando los de mayor vigor y mejores características, que superaron a los mejores genotipos de las progenies híbridas $F_{1}$ de cruzas dialélicas paralelamente desarrolladas.

La alta variabilidad de las progenies de polinización libre también se habría incrementado por el mayor número de progenitores que intervinieron (23 a 51), a diferencia que en las cruzas dialélicas solamente intervinieron de 10 a 15 progenitores.

Finalmente, puede concluirse que el método de polinización libre en el desarrollo de cultivares nativos tetraploides andigena es eficiente, y es más simple y económico que los métodos dialélicos completos. 


\section{CONCLUSIONES}

En la determinación de la estabilidad fenotípica de cuatro caracteres de 57 clones $\mathrm{F}_{1}$ bajo seis ambientes se encontró que:

1. El clon P21-38-0 de polinización libre es ESTABLE en 3 caracteres simultáneamente: rendimiento, tamaño de tubérculos y gravedad específica.

2. Los clones P09-07-8, P13-59-9, Pl1-01-0 y P02-01-0 son ESTABLES en 2 caracteres simultáneamente: en rendimiento y tamaño, en rendimiento y número, y los dos últimos, ambos en número y gravedad específica.

3. Once clones $\mathrm{F}_{1}$ dialélicos y 11 clones $\mathrm{F}_{1}$ de polinización libre son ESTABLES en un solo carácter.

4. Los clones P21-38-0, P09-07-8, P01-11-0 y P02-01-0 serán nominados como nuevos cultivares nativos tetraploides andigena, para su utilización por los agricultores.

5. En el desarrollo de nuevos cultivares nativos tetraploides andigena, el método de polinización libre es genéticamente eficaz, más simple y económico que los métodos dialélicos completos.

\section{REFERENCIAS BIBLIOGRÁFICAS}

1. Eberhart, S.A.; Russell, W.L. 1966. Stability parameters for comparing varieties. Crop Sci. 6:36-40.

2. Finlay, K.W.; Wilkinson, G.N. 1963. The analysis of adaptation in a plant-breeding programme. Australian J. Agr. Res. 14:742-754.

3. Freeman, G.H. 1973. Statistical methods for the analysis of genotypeenvironment interactions. Heredity 31:339-354.

4. Golmirzaie, A.M. 1988. Comparison of hybrids with different openpollinated (OP) generations of true potato seeds (TPS) from agronomic characters. Amer. Potato J. 65(8):480.

5. Hill, J. 1975. Genotype-environment interactions - a challenge for plant breeding. J. Agric. Aci., Camb. 85:477-493.

6. Javier, T.G. 1990. Selección de clones $F_{1}$ de cruzas dialélicas y de polinización libre en papas nativas. Artículo Inédito. Proyect de T.T.A. FUNDEAGRO e INVESTIGADOR. Huancayo, Perú. 23 p. 
7. Javier, T.G. 1991. Selección de clones $F_{1}$ de cruzas dialélicas y de polinización libre en papas nativas. Informe Final. Convenio FUNDEAGRO e INVESTIGADOR. Huancayo, Perú. 26 p.

8. Mendoza, H.A.; Marca, J.L. 1986. Performance of selfed, open-pollinated, and hybrid progenies in a Solanum tuberosum ssp. andigena population. Amer. Potato J. 63(8):444.

9. Tai, G.C.C. 1971. Genotypic stability analysis and its application to potato regional trials. Crop Sci. 11:184-190.

10. Tai, G.C.C.; Young, D.A. 1980. A biometric model for predicting the performance of potato clones in different environments. Report of the Planning Conference CIP. Lima, Perú. pp. 184-203. 